\title{
Interactive comment on "Snow cover variations across China from 1952-2012" by Xiaodong Huang et al.
}

\section{Anonymous Referee \#1}

Received and published: 22 October 2019

This manuscript characterizes snow cover variability and trends across three regions of China through the analysis of the surface snow depth observation network. While the subject is relevant to The Cryosphere and represents a potentially important contribution to the assessment of regional snow cover trends, there are some critical shortcomings.

1. The end date of 31 December 2013 is problematic. It makes more sense to use the end of a snow season or hydrological year. Small point, but because the complete 2012-2013 season was available for analysis, should the title not use the year range 1952-2013? More importantly, why was the seemingly arbitrary decision made to only utilize data through 2013, which means the reported trends do not include the six most recent winters. This means the reported trends have reduced relevancy and timeliness.

Printer-friendly version

Discussion paper 
As noted below, there were some extreme snow events in China during the 2009-2012 time period, so it would be interesting to see if these strong anomalies continue to emerge between 2013 and 2019.

2. Lines 85-92: It's not clear how time series of varying length at individual measurement locations were utilized in the analysis. Do the map figures (e.g. Figures 2, 4, and 6) show all stations with at least 10 years of data as noted on line 86 ? If so, does this mean there are different start and end dates for individual stations in these figures, or do all points over the same time period? Do some points represent trends calculated over only 10 years of data? In the figures which show regional averages (e.g. Figures 3,5 , and 7 ) how was variability in station time series length accounted for in the averaging procedure? More details on how the snow data were handled is needed in the first part of Section 2.1.

3. Some of the analyzed snow variables are consistent with typical metrics of assessing snow cover, such as maximum snow depth, snow cover days, snow onset, snow end days, etc. but some of the snow variables are unusual and lack a clear physical driver that can be related to climate variability. I suggest dropping annual average snow depth and cumulative snow depth. In the case of snow onset date (SOD) and snow end date (SED), do these mark the beginning and end of continuous snow cover, or do they include ephemeral snow cover events (such as the first time snow accumulates, even if it melts again before continuous snow cover begins)?

4. Section 2: the text describing Mann-Kendall and linear trends in parts (a) and (b) is not easily readable - it's essentially a series of equations which describe fairly straightforward statistical techniques. Sections (c) and (d) however provide only very short descriptions of more complicated techniques. Should the wavelet analysis and structural equation modeling be retained in the paper (see comment \#8 below) the text in parts (c) and (d) must be expanded, while the readability of parts (a) and (b) should be improved. 
5. Section 3.1: I think most readers will not be clear on the terms UF and UB as utilized in the text. The definition of UF can be discerned from Section 2.1, but this is not the case for UB.

6. Figures 3 and 5: the strength of these trends seem to be driven strongly by the early and late years in the time series. Without a handful of large anomalies during the first five years and the last five years, the trends appear to be near zero. Can some comments be added on the influence of these outliers at the beginning and end of the time series?

7. I'm confused by the determination of some of the break points in the time series. For example, in Table 2 - how can some regions and variables have break points in consecutive years (such as 1956 and 1957)?

8. Many of the overall trends are quite modest (e.g a decrease in snow cover days of 0.1 days per decade, which translate to not even a full day over the 70-year period of record). The lack of trends is itself not an issue - it's still useful to report this in the literature given the long time period. But with the lack of strong trends evident in the Mann-Kendall analysis, I don't believe the wavelet analysis and structural equation modeling are warranted. My suggestion would be to remove this analysis - Figures 8 and 9 are difficult to interpret and don't add much insight. Instead, some additional analysis could add more value and innovation to the paper. I was left wondering two things: -Are these trends consistent with satellite-derived data such as the NOAA snow chart climate data record (https://climate.rutgers.edu/snowcover/index.php) which extend back to 1977? -Can snow cover trends over China be attributed to temperature and/or precipitation trends? In general, it appears the snow cover season is getting shorter (Figure 7) but the snow depth is increasing (Figure 3). How do the spatial patterns of seasonal temperature changes and precipitation change (including the proportion of snow versus rain) compare to the station observations of snow?

Printer-friendly version

A couple minor comments: -Line 17: the term 'jump points' is used here and throughout 
the manuscript, but I think 'break points' is a more appropriate term -Figure 1: Given the emphasis placed on elevation for some of the interpretation, can shading be added to this figure to show elevation?

The manuscript also requires a thorough edit for grammar, English usage, and word choice. Edits of this nature were too numerous to identify individually in this review.

Interactive

comment

Interactive comment on The Cryosphere Discuss., https://doi.org/10.5194/tc-2019-152, 2019. 